\title{
Study on Identification of the Pressure- Resistant Bacteria Isolated from Coconut Puree and its Lethal Effect with Combined Mild Temperature and Ultrahigh Pressure
}

\author{
Bin Wan,Zhen-hua Duan*,Shan-shan Luo \\ College of Food Science and Technology \\ Hainan University \\ Haikou, China \\ dzhwj1@hotmail.com(corresponding author)
}

\author{
Wei-wen Duan,Fei-fei Shang \\ College of Food Science \\ Hezhou University \\ Hezhou, China \\ dww@126.com
}

\begin{abstract}
This research based on fresh coconut and the coconut puree was conducted with the ultra-high pressure $(500 \mathrm{MPa} / 10 \mathrm{~min})$. Two strains of pressure-resistant bacteria in coconut puree were screened out and identified as Leuconstoc mesenteroides subsp mesenteroides and Leuconstoc mesenteroides subsp dextranicum When comparing with constant and intermittent pressure treatments. Treatment with the intermittent pressure of $300 \mathrm{MPa}-600 \mathrm{MPa}$ can greatly improve the lethal effect of pressure-resistant bacteria. The fatality rate on $L$. mesenteroides subsp mesenteroides and $\mathrm{L}$. mesenteroides subsp dextranicum were $\mathbf{9 2 . 6 \%}$ and $\mathbf{8 9 . 5 \%}$. Lethality goes up significantly along with the temperature's rising. At $65^{\circ} \mathrm{C}$,lethality of L.mesenteroides subsp mesenteroides and L.mesenteroides subsp dextranicum were $\mathbf{9 8 . 9 \%}$ and $98.2 \%$.
\end{abstract}

Keywordt coconut puree; pressure-resistant bacteria; identification; ultrahigh pressure;lethality

\section{INTRODUCTION}

Cocos nucifera L. belong to Palmaceae in Cocos. It is mainly one of the perennial woody oil crops and energy crops in tropical area. Coconut is a tropical hi-light plant, which are widely distributed in Asia, Africa, Oceania and Americas, especially in the equator distribution in coastal area. Coconut producing area are mainly distributed in the southeast coast of Hainan island in China, which the area and production about 80 percent of the total amount of the country. The coconut is mainly composed of exocarp, mesocarp, endocarp, seed coat, coconut, coconut water and other parts. Coconut, called the solid endosperm, is the main component of coconut.

Fresh coconut contains $35.2 \%$ of fat, $3.8 \%$ of protein and $40 \%$ of moisture content, and a small amount of VB1, $\mathrm{VA}, \mathrm{VC}, \mathrm{VE}, \beta$-carotene and polyphenols, which have the benefit of spleen and stomach, prevent hyperlipidemia, protect the cardiovascular system [1-2].The coconut had wasted in a certain extent because of the large, heavy and high proportion of coconut, added to feeding and carrying is more inconvenient. Turning coconut into puree will promote the fresh-keeping of coconut and is more advantageous to the consumer demand based on original nutritional quality. Coconut is perishable for the inevitably infection by microorganisms in product processing. The study found that only rely on the separate ultrahigh pressure processing is difficult to achieve the sterilization requirements of coconut puree [3].In the process of storage, With the residual barotolerant microbes multiply, the nutrient had decreased and the shelf life had shorten. Therefore, studying for barotolerant microbes of coconut puree is of great significance on coconut meat storage and fresh keeping. This paper adopted the ultra-high pressure $500 \mathrm{MPa} / 10 \mathrm{~min}$ to deal with the coconut puree and the barotolerant microbes in coconut puree were screened out and identified by morphology, physiology, and molecular biology (16SrDNA) method. At the same time, the lethal effect of the combined mild temperature and ultrahigh pressure treatment on the barotolerant microbes was studied. To improve the theoretical basis for the sterilization technology of coconut puree.

\section{MATERIALS AND METHODS}

\section{A. Materials and reagents}

Coconut were bought in the wholesale market of Newport Bridge in Haikou, selected the watering sound small, the shell color deeper and the mature moderate of coconut.

Peptone bacteriological and Beef extract were purchased from Guangdong central Kai Microbial Technology Company Limited; Anhydrous glucose, Agar bacteriological, D (+) galactose, glucose, mannose, xylose and maltose, sucrose and arabinose, were bought from the Pharmaceutical Group Chemcial Reagent Company Limited.

Biological reagents, including bacterial genome DNA extraction kit, PCR recycling kit and purification, dNTP Mix, Taq buffer, goldview, DL2000, DNA Ladder Mix, were provided by Tiangen Reagent Company.

\section{B. The configuration of the main culture medium}

Nutrient AGAR medium (NA): peptone10.0g, beef extract3.0g, sodium chloride5.0g, Agar $17.0 \mathrm{~g}$, add distilled 
water to $1000 \mathrm{~mL}$, dissolved and adjusted the $\mathrm{pH}$ to 7.2 , packaging sterilization $\left(121^{\circ} \mathrm{C}, 20 \mathrm{~min}\right)$.

Nutrient broth (NB) medium: peptone $10.0 \mathrm{~g}$, beef extract3.0g, distilled water $1000 \mathrm{~mL}$. Adjusted $\mathrm{pH}$ to 7.2 , packaging sterilization $\left(121^{\circ} \mathrm{C}, 20 \mathrm{~min}\right)$.

Plate count AGAR (PCA): tryptone5.0g, yeast extract $2.5 \mathrm{~g}$, glucose $1.0 \mathrm{~g}$, Agar $15.0 \mathrm{~g}$, distilled water 1000 $\mathrm{mL}$, dissolved and adjusted the $\mathrm{pH}$ to $7.0 \pm 0.2$, packaging sterilization $\left(121^{\circ} \mathrm{C}, 20 \mathrm{~min}\right)$.

\section{Instruments and equipment}

HPP. L3-600/0.6 ultrahigh pressure equipment, Tianjin Huatai Senmiao Company Limited; SW-CJ-1 type fd bench, Suzhou Antai air technology company., LTD;DZ$500 / 2$ s type vacuum packaging machine, Shandong Zhucheng leading machinery co.,LTD; IKA A11Basic analysis using grinding machine, Germany IKA company; AL-204 electronic analytical balance, mettler Toledo instrument (Shanghai) co., LTD.; ZEALWAYGR60DA autoclave, Xiamen micro instrument co.,LTD.;LRH-150-B biochemical incubator, medical apparatus and instruments factory in Guangdong province; Nikon ECKIPSE Ci-s/CiL microscope, Nanjing HengQiao instrument co.,LTD.; RDY-SP1Z nucleic acid electrophoresis apparatus, Beijing RongYang classic science and technology co., LTD.A200 gene amplification, Hangzhou lang base scientific instrument co., LTD.;JY04S-3C gel imaging system, Beijing Oriental electrophoresis equipment co., LTD.

\section{Test method}

1) The coconut puree of ultrahigh pressure processing

The coconut puree with the vacuum packaged was placed in the pressure medium of the ultra-high pressure vessel,at $500 \mathrm{MPa}$ under $10 \mathrm{~min}$. Each sample was carried out for three parallel tests[3].

2) The separation and purification of barotolerant microbes

The coconut puree with processing of ultra-high pressure were homogenized according to the GB47892010. Drawing the $200 \mathrm{uL}$ homogenate in the PCA medium plate, coating by triangle glass rod, and each dilution gradient of homogeneous liquid coated three plates, cultivated $24-48 \mathrm{~h}$ at $37^{\circ} \mathrm{C}$. The bacterial colony was picked on NA medium plate by the sterile toothpicks, crossed and purified repeatedly, the single colony was obtained. The number of the obtained strains was kept at $4^{\circ} \mathrm{C}$ for reserve [4].

3) Morphology observation of barotolerant microbes

Colony morphology: observed and recorded each colony morphology of nutrient AGAR plate by means of macroscopic. Including colony size, shape, convex surface, edge conditions, colony morphology, surface gloss, color of the colony and colony transparent degree, etc.

Cell form: the strains were separated by the gram staining, bacteria morphology observed by optical microscope and record observations.

4) Physiological and biochemical characteristics of the experiment

Determination of physiological and biochemical characteristics in accordance with the "berger bacteria identification manual" eighth edition (Buchanan et al,1984) and "common bacteria system identification manual".
5) Molecular identification of barotolerant microbes

16SrDNA amplified primers were common primers for bacteria, the forward primer was P1: 5'-AGAG TTT GAT CCTGGTCAGAACGCT-3,40pmol; the reverse primer was P6: 5'-T ACG GCT ACC TTG TTA CGA CTTCACCCC-3',40pmol, the primer was synthesized by Sangon biological engineering. Using $25 \mathrm{~mL}$ amplification system for PCR amplification [5-7].

6) Sequencing and analysis

After the purification, transformed, the PCR product was obtained and sent to Sangon biological engineering for sequencing. The Blast analysis of the sequence and the GenBank database showed that the results of identification of 16S rDNA [8].

\section{E. The lethal effect of different pressure mode on barotolerant microbes}

1) The preparation of bacterium suspension

The barotolerant microbes was inoculated in a sterile nutrient broth medium, cultivated 24-48h at $30^{\circ} \mathrm{C}, 200 \mathrm{r} / \mathrm{min}$. A small amount of activated bacteria was absorbed and the blood cell counting plate was used to carry the microscope, the initial concentration of the bacteria suspension was about $10^{8} \mathrm{cfu} / \mathrm{mL}$. Drawing the $10 \mathrm{~mL}$ bacteria suspension in the sterile polyethylene bags, vacuum packaging [4].

2) The treatment of different pressure mode on barotolerant microbes

Constant pressure treatments: the bacteria suspension with the vacuum packaged was placed in the pressure medium of the ultra-high pressure vessel, respectively in $300 \mathrm{MPa}$ and $600 \mathrm{MPa}$ pressure under $10 \mathrm{~min}$ at $30^{\circ} \mathrm{C}$.

Intermittent pressure treatments: the bacteria suspension with the vacuum packaged was placed in the pressure medium of the ultra-high pressure vessel, started with a lower pressure $\left(300 \mathrm{MPa}\right.$ at $\left.30^{\circ} \mathrm{C}\right)$ treatment for $5 \mathrm{~min}$, followed by a higher pressure $\left(600 \mathrm{MPa}\right.$ at $\left.30^{\circ} \mathrm{C}\right)$ for another $5 \mathrm{~min}$.

\section{F. The lethal effect of different processing temperature on barotolerant microbes}

The bacteria suspension with the vacuum packaged was placed in the pressure medium of the ultra-high pressure vessel, At the pressure of $300 \mathrm{MPa}-600 \mathrm{MPa}$, respectively in $35^{\circ} \mathrm{C}, 45^{\circ} \mathrm{C}, 55^{\circ} \mathrm{C}, 65^{\circ} \mathrm{C}$ under $10 \mathrm{~min}$, which pressure time of $300 \mathrm{MPa}$ and $600 \mathrm{MPa}$ was $5 \mathrm{~min}$.

\section{G. The determination of the fatality rate}

According to GB4789.2-2010, microbial counts were determined by the method of plate coating, and the fatality rate was calculated [9].

\section{RESULTS AND ANALYSIS}

\section{A. Isolation and purification of barotolerant microbes}

The coconut puree with Vacuum packed was treatment for $500 \mathrm{MPa}$ under $10 \mathrm{~min}$ at $30^{\circ} \mathrm{C}$, the homogeneous liquid was coated in PCA medium, and cultivated 24-48h at $37^{\circ} \mathrm{C}$. The morphology of colonies was separated from 
the surface of the medium, and the colonies were numbered WS1 and WS2 respectively. Colony morphology, cell shape as shown in figure 1, figure 2

Figurel and figure 2 showed that the strain WS1 colonies were spherical, and the diameter was $0.5-1 \mathrm{~mm}$, the surface of the colony was sticky and rose, the color was white. The strain WS2 colonies were spherical, and the diameter was $1-2 \mathrm{~mm}$, the surface of the colony was smooth and rose, the color was hoary. The strain WS1 and WS2 were gram-positive bacteria and the cell morphology were in pairs and streptococcal by microscopic examination of smearing and gram staining.

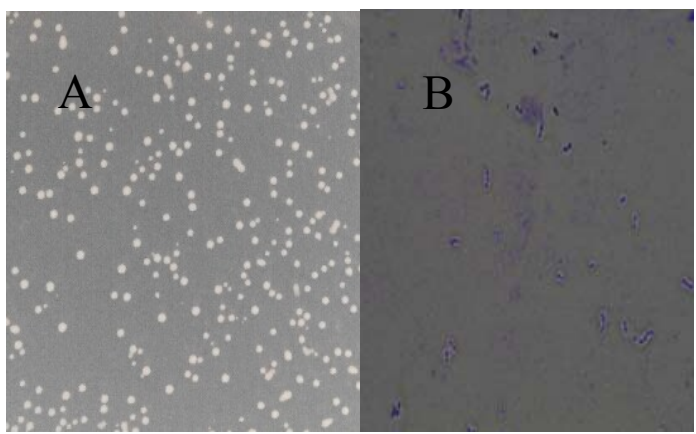

Figure 1. Photographs of colonies(A) and cells(B) of the strain WS1

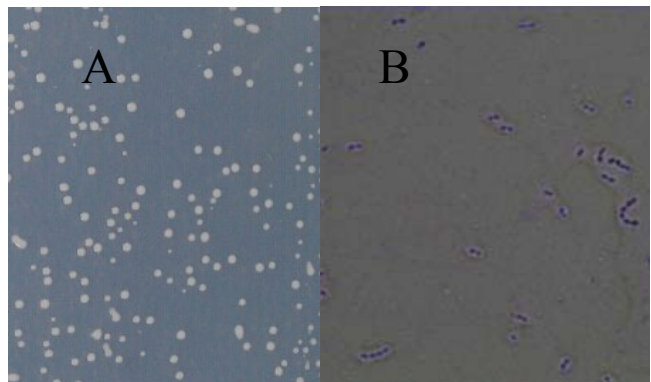

Figure 2. Photographs of colonies(A) and cells(B) of the strain WS2

\section{B. Physiological and biochemical characteristics of barotolerant microbes}

The physiological and biochemical identification of the strain WS1 and WS2 was carried out by the physiological and biochemical test of the Berger's bacterial identification manual. The results were shown in Table 1.

From the table 1, the catalase of the strain WS1 and WS2 were all negative and the strains were non acidification, but freezing milk and fermenting galactose, fructose, maltose, mannose, xylose, sucrose and glucose. The glucan was produced in the identification of the culture medium. The arabinose was fermented by the strain WS1 that it can be produced the acid, and the strain WS2 was not. Through the comparison of Berger's bacterial identification manual, the WS1 and WS2 were identified as Leuconostoc spp.
TABLE1 RESULT OF BIOCHEMICAL AND PHYSIOLOGICAL IDENTIFICATION OF WS1 AND WS2

\begin{tabular}{ccc}
\hline project & WS1 & WS2 \\
\hline catalase & - & - \\
litmus milk & - & - \\
galactose & + & + \\
fructose & + & + \\
maltose & + & + \\
mannose & + & + \\
xylose & + & + \\
sucrose. & + & + \\
glucose & + & + \\
arabinose & + & - \\
glucan & + & + \\
\hline Comment:“+”positive reaction; “_”negative reaction
\end{tabular}

\section{The sequences analysis of $165 r$ DNA}

\section{1) The extraction of genomic DNA}

Genomic DNA was extracted from bacterial genomic DNA rapid extraction kit and detected by $1 \%$ gaerose gel electrophoresis. The results were shown in Figure 3. There is no impurity in the rubber hole, the band has no interference, the length of the DNA fragment was about $1500 \mathrm{bp}$, and the DNA was better that can be amplified by PCR.

\section{$\begin{array}{lll}1 & 2 & \mathbf{M}\end{array}$}

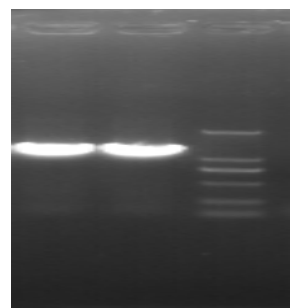

Figure 3. The extraction of genomic DNA of strain WS1 and WS2 M: DL2000 marker 1-2: The extraction of genomic DNA of strain WS1 and WS2

2) The amplification and detection of $16 S \mathrm{rDNA}$

The results of PCR amplification were showed in Figure 4, the length of the amplified sequence was $1500 \mathrm{bp}$, with the size of expected to, After the purification, transformed, the product was obtained and sent to Sangon biological engineering for sequencing.

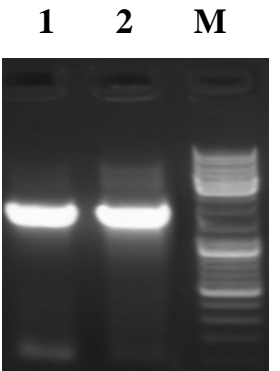

Figure 4. PCR amplification of 16S rDNA of strain WS1 and WS2 M: DNA Ladder Mix make 1-2: Mean PCR result of strain WS1 and WS2 
3) Phylogenetic and homology analysis of $16 \mathrm{SrDNA}$ sequences

The Blast analysis of the sequence and the GenBank database showed that the sequence of 16SrDNA of the strain WS1similarity of the Leuconstoc mesenteroides subsp mesenteroides reached $99 \%$ in GenBank. The strains of WS2 and Leuconstoc mesenteroides subsp dextranicum of 16 SrDNA sequence similarity as high as $99 \%$.According to the homology analysis results, the strain WS1 was identified as Leuconstoc mesenteroides subsp mesenteroides and the strain WS2 was Leuconstoc mesenteroides subsp dextranicum.

Two strains of barotolerant microbes in coconut puree were screened out and identified as Leuconstoc mesenteroides subsp mesenteroides and Leuconstoc mesenteroides subsp dextranicum by morphological features, physiology and molecular biology (16SrDNA) method. Molecular phylogenetic tree based on $16 \mathrm{Sr}$ DNA sequence WS1 and WS2 were showed in Figure 5

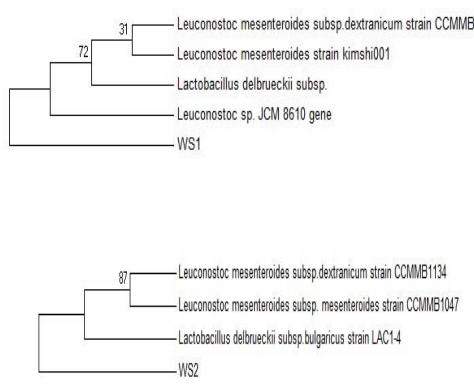

Figure 5. Molecular phylogenetic tree based on 16Sr DNA sequence WS1 and WS2

\section{The lethal effect of different pressure mode on barotolerant microbes}

Figure 6 showed that different pressure mode on Leuconstoc mesenteroides subsp mesenteroides (A) and dextranicu(B) have better lethal effect. Lethal effect of intermittent preesure treatments on strains is superior to contant pressure treatment under the same condition, when comparing with constant and intermiteent pressure treatments. Treatment with intermittent pressure of 300$600 \mathrm{MPa}$ can greatly improve the lethal effect of compression strain. The fatality rate on Leuconstoc mesenteroides subsp mesenteroides and Leuconstoc mesenteroides subsp dextranicum were $92.6 \%$ and $89.5 \%$.

Analysis of the mechanism that is: The stress of the cell membrane and the cell wall were strengthen from low to high pressure and the process of booster and unloading repeatedly, which made the cell more vulnerable and destroyed [10].Therefore, choosing the combination of intermittent pressure as a sterilization way for ultra-high pressure treatment on coconut puree.
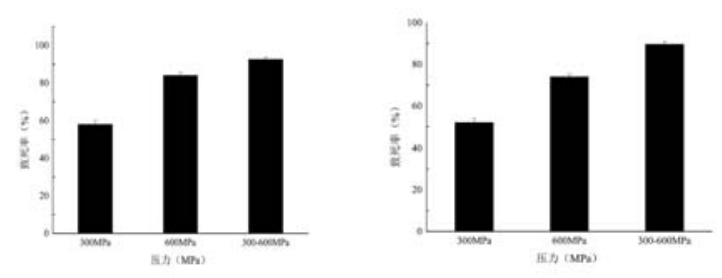

Figure 6. The lethal effect of different pressure mode on Leuconstoc mesenteroides subsp mesenteroides (A)and dextranicum(B)
E. The lethal effect of different processing temperature on barotolerant microbes

In coordination with temperature were $35^{\circ} \mathrm{C}, 45^{\circ} \mathrm{C}$, $55^{\circ} \mathrm{C}, 65^{\circ} \mathrm{C}$ and combined with the $300 \mathrm{MPa}-600 \mathrm{MPa}$ pressure on Leuconstoc mesenteroides subsp mesenteroides (A) and dextranicu(B),the results as showed in Figure7. Lethality goes up significantly along with the temperature increasing and when the temperature is above $55^{\circ} \mathrm{C}$, the lethality increases smoothly. At 65 , lethality of $L$. mesenteroides subsp mesenteroides and $L$. mesenteroides subsp dextranicum were $98.9 \%$ and $98.2 \%$. Kaletunc researched that the cell structure of Leuconostoc spp has changed, when the processing of $500 \mathrm{MPa}, 35^{\circ} \mathrm{C}, 15 \mathrm{~min}$. Such asbeaded structure of Leuconostoc spp. was destroyed and the part of the cell surface forming processes, besides, the precipitation was formed within cells and the destruction of cell membrane resulted in the decrease of $\mathrm{Mg}^{2+}$ concentration [11].Thus, choosing the $35^{\circ} \mathrm{C}, 45^{\circ} \mathrm{C}, 55^{\circ} \mathrm{C}$ as processing temperature for ultra-high pressure treatment on coconut puree.
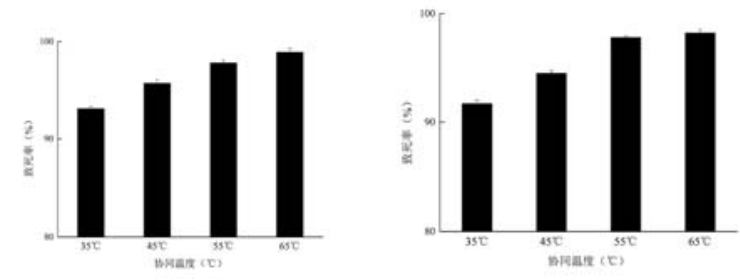

Figure 7. The lethal effect of different processing temperature on Leuconstoc mesenteroides subsp mesenteroides (A)and dextranicum(B)

\section{DISSCUSSION}

This article isolated the two strains of barotolerant microbes from the coconut puree, which was treated by ultra-high pressure. The two strains were L.mesenteroides subsp mesenteroides and L.mesenteroides subsp dextranicum. Research found that the L.mesenteroides subsp and Weissella viridescens were typical barotolerant microbes in meat products [12]. In addition, there were a lot of studies on the barotolerant microbes. Such as, Feng research found that the barotolerant microbes of salted dunk including Staphylococcus warneri, Staphylococcus epidermidis, Enterococcus faecalis and Bacillus cereus, which the corrosion capability of Bacillus cereus was the strongest [13]. Li also found that Bacillus pumilus of pickle had a strong compressive ability.

Lethal effect of intermittent preesure treatments on strains is superior to contant pressure treatment under the same condition, when comparing with constant and intermiteent pressure treatments. Treatment with intermittent pressure of $300 \mathrm{MPa}-600 \mathrm{MPa}$ can greatly improve the lethal effect of compression strain. The fatality rate on Leuconstoc mesenteroides subsp mesenteroides and Leuconstoc mesenteroides subsp dextranicum were $92.6 \%$ and $89.5 \%$. Lethality goes up significantly along with the temperature increasing and when the temperature is above $55^{\circ} \mathrm{C}$, the lethality increases smoothly. At 65 , lethality of $L$. mesenteroides subsp mesenteroides and L. mesenteroides subsp dextranicum were $98.9 \%$ and $98.2 \%$. Therefore, Choosing 
the combined mild temperature and ultrahigh pressure intermittent sterilization of coconut puree

\section{CONCLUSION}

1. Two strains of barotolerant microbes in coconut puree were screened out and identified as Leuconstoc mesenteroides subsp mesenteroides and Leuconstoc mesenteroides subsp dextranicum by morphological features, physiology and molecular biology (16SrDNA) method.

2. Combined mild temperature and ultrahigh pressure intermittent of coconut puree has significant sterilization effect. And the stress effect was strengthen from intermittent pressure way which had a process of step-up and step-down. The greater the pressure, the higher the temperature, the stronger the lethal effects

\section{ACKNOWLEDGMENT}

This work was financially supported by the Hainan Province Natural Science Foundation, China (20153078).

\section{REFERENCES}

[1] WAN, B. \& DUAN, Z.H.2014. Effect of Combined Mild Temperature and Ultrahigh Pressure on Quality and Sterilization of Coconut Puree. Modern Food Science and Technology30(9):222229.

[2] JIN,B.\&HUANG, M.F.2013. Enzymatic hydrolysis of coconut protein and antioxidant activity of the hydrolysates. Modern Food Science and Technology (8):1826-1831.

[3] KANG, R. \& DUAN, Z.H.2013. Effect of ultra-high pressure treatment on fresh-keeping of coconut puree .Science and Technology of Food Industry34(7): 134-136
[4] LI, X.M. 2012.Inactivation of Baroduric Bacteria Isolated by High Hydrostatic Pressure in Pickle of Sichuan. Sichuan Agricultural University

[5] XU,F. \&LIU,Y. 2011.Isolation of a Bacillus Str ain from Packaged Pleurotus geesterani Fruit Bodies Pr ocessed Using Super High Pressure Technology. Journal of edible fungi18(1):59-62

[6] LONG, X.F\&CHEN W.J. 2014.Quality change of coconut meat under low temperature andisolation and identification of specific spoilage organism. Agricultural Sciences of Guangdong 9:108-112

[7] YU, K.W. \&SANG W.G. 2010.Isolation, purification and characterization of microbes from rice cake.Science and Technology of Food Industry31(2):167-169

[8] DUAN, A.L \&LEI, Y.S.2013. SUN Xiang-yu,et al. Molecular Identification and rDNA-ITS Sequence Analysis of Fungal Pathogens in Kiwifruit During Storage. Scientia Agricultura Sinica46(4):810-818.

[9] LIU, J. \& LIN, Y.2008.Effects of high-hydrostatic pressure on inactivation of Bacillus subtilis spores. Industrial microorganism38(4):35-38

[10] Mohamed,H.M.H \&Diono, B.H.S.2012. Structural Changes in Listeria Monocy-Togenes Treated With Game Radiation,Pulesed Electric Field and Ultra-High Pressure.J Food Safe32(1):66-73

[11] Kaletunc, G. \&Lee, J. 2004.Evaluation of Structural Changes Induced by High Hydrostatic Pressure in Leuconostoc mesenteroide. Appl Environ Microbiol70(2):1116-1122

[12] WEN,S.Y. \& HAN, Y.Q.2012. Combined effect of ultra high pressure and Nisin on inactivation of spoilage pressure-resistant bacteria isolated from smoked cooked ham.Science and Technology of Food Industry01:59-62.

[13] FENG, Y.L.\&WANG,H.H. 2014.Study on identification and basic characteristics of the pressure-resistant bacteria isolated in Nanjing water-boiled salted duck. Science and Technology of Food Industry 18:167-170 